\title{
CORE POLARIZATION EFFECTS ON ELECTROMAGNETIC TRANSITION OPERATORS
}

\author{
A. E. L. DIEPERINK and P. J. BRUSSAARD \\ Fysisch Laboratorium van de Rijksuniversiteit, Utrecht, Netherlands
}

Received 1 November 1968

\begin{abstract}
Effective proton and neutron charges have been calculated for the E2 operator in the sd and $\mathrm{fp}$ shells in first-order perturbation theory. Second-order corrections have been calculated for the M1 operator and $f t$ values for $A=39$ and $A=41$ nuclei. The realistic Tabakin interaction has been employed.
\end{abstract}

\section{Introduction}

In the shell-model description of spherical nuclei one usually selects a small number of single-particle orbits near the Fermi surface. These valence orbits span the model space; energy spectra can be obtained by diagonalizing an effective interaction in this space, i.e., the possible excitations of particles from lower-lying orbits forming the core are not taken into account explicitly. The effective interaction can be parametrized in various ways. For such phenomenological forces, it is assumed that all core effects are absorbed in the adjustable parameters.

However, there exists a more fundamental approach to the problem of the nuclear interaction in nuclei. From the scattering data of free nucleons in the energy range 0-350 MeV, so-called realistic potentials have been derived. Of course, these bare realistic nucleon-nucleon interactions are not expected to be used directly in a spectroscopic calculation for finite nuclei, e.g. one should correct for the finite size of the configuration space used. Numerous calculations with realistic forces have been performed. It was found in these cases ${ }^{1,2}$ ) that a significant renormalization of the two-body interaction results from polarization effects of the core. This renormalization considerably improves the agreement with the experimental energies.

Similarly the electromagnetic properties that are ascribed to the valence nucleons must be renormalized due the presence of the core nucleons. The shell-model predictions of electromagnetic moments and transition rates in general do not very well reproduce the experimental values.

Core-polarization effects on one-body operators have been investigated mostly with phenomenological nucleon-nucleon forces, which are designed to eliminate the explicit influence of the core.

Since one now has realistic forces which have been applied rather successfully, it is desirable to investigate quantitatively the effect of core polarization on one-body operators with these forces. 
Therefore, in this paper we shall consider the effect of core polarization on onebody operators for nuclei in the $A=39-41$ region. We shall apply the Tabakin interaction ${ }^{3}$ ) that thas been used in several shell-model calculations ${ }^{2,4,5}$ ) previously.

It has been found ${ }^{2,6}$ ) that this interaction is not smooth enough for first-order terms to suffice. However, if second-order corrections are included, the matrix elements are very similar ${ }^{2}$ ) to those of the $G$-matrix calculated for the hard-core Hamada-Johnston potential.

In sect. 2, the corrections to electromagnetic transition rates are derived in firstorder perturbation theory; the results are applied to derive effective neutron and proton charges for elect 1 ic quadrupole moments and transitions. In sect. 3, the results are presented of second-order perturbation theory applied to magnetic dipole moments and transitions as well as to $f t$ values. Concluding remarks are made in sect. 4.

\section{Effective-charge operators in $\boldsymbol{A}=39-41$ nuclei}

It is well known that the experimental reduced transition probabilities $B(E 2)$ for transitions in nuclei consisting of closed shells plus or minus one nucleon can deviate considerably from the single-particle predictions; e.g. the $B(\mathrm{E} 2)$ value for the transition ${ }^{7}$ ) from the lowest $J^{\pi}=\frac{3}{2}^{-}$state to the $J^{\pi}=\frac{7}{2}-$ ground state in ${ }^{41} \mathrm{Ca}$ is found to be two times larger than the single-proton $2 \mathrm{p}_{\frac{1}{2}} \rightarrow 1 \mathrm{f}_{\frac{1}{2}}$ value if harmonic-oscillator wave functions are used. The ground state electric quadrupole moments ${ }^{8}$ ) of ${ }^{39} \mathrm{~K}$ and ${ }^{40} \mathrm{~K}$ are also approximately a factor of 1.5 larger than a pure $1 \mathrm{~d}_{\frac{3}{3}}$ hole state would yield. In some recent shell-model calculations ${ }^{9,10}$ ) effective neutron and proton charges have been introduced in order to fit the experimental $B(E 2)$ values. These calculations indicate that effective charges of approximately $0.5 e$ both for the neutron and the proton are required to fit the experimental $B(E 2)$ values. We shall investigate whether core polarization can account for these numbers.

\subsection{FORMALISM}

For brevity a direct-product notation will be used, where Greek symbols denote the quantum numbers in configuration space and in isospace, e.g. $(-1)^{x}=(-1)^{j_{\alpha}+t_{\alpha}}$.

Double-bar matrix elements will be used according to the convention of ref. $\left.{ }^{11}\right)$. Matrix elements reduced with respect to both configuration space and isospace are denoted by triple-bar matrix elements.

To first order in the interaction, the corrections to the reduced single-particie matrix elements of an electromagnetic transition operator can be represented by diagrams la and $1 \mathrm{~b}$ (exchange diagrams are omitted). The contribution of these first-order diagrams to the reduced matrix elements

$$
\left\langle\alpha_{\mathrm{f}}||\left|\boldsymbol{\Omega}^{(A)}\right| \mid \alpha_{\mathrm{i}}\right\rangle
$$

of the single-particle operators $\Omega^{(\Lambda)}(\Lambda$ denotes rank $k$ in configuration space and 
rank 0 or 1 in isospace) is given by

$$
\begin{array}{r}
\Delta^{(1)}\left\langle\alpha_{\mathrm{f}}||\left|\boldsymbol{\Omega}^{(\Lambda)}\right||| \alpha_{\mathrm{i}}\right\rangle=\sum_{\alpha_{1} \alpha_{2} \Gamma} \frac{-1}{|\Delta E|}(-1)^{\alpha_{2}+\alpha_{1}+\Gamma}(2 \Gamma+1)\left\{\begin{array}{lll}
\alpha_{\mathrm{i}} & \alpha_{\mathrm{f}} & \Lambda \\
\alpha_{1} & \alpha_{2} & \Gamma
\end{array}\right\}\left(\left(1+\delta_{1 \mathrm{f}}\right)\left(1+\delta_{2 \mathrm{i}}\right)\right)^{\frac{1}{t}} \\
\times\left\langle\alpha_{\mathrm{i}} \alpha_{2} ; \Gamma|V| \alpha_{\mathrm{f}} \alpha_{1} ; \Gamma\right\rangle\left\langle\alpha_{2}\left\|\left|\boldsymbol{\Omega}^{(\Lambda)} \|\right| \alpha_{1}\right\rangle\right.
\end{array}
$$

Here $\left\langle\alpha_{a} \alpha_{b} ; \Gamma|V| \alpha_{c} \alpha_{d} ; \Gamma\right\rangle$ represents an antisymmetric and normalized two-body matrix element of the nucleon-nucleon interaction $V$, where the two nucleons are

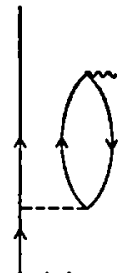

(a)

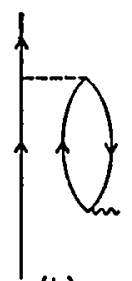

(b)

Fig. 1. First-order single-particle renormalization diagrams.

coupled to definite spin and isospin $\Gamma$. For diagram la the index $\alpha_{1}$ runs over particle states and $\alpha_{2}$ over hole states $\left(\Delta E=\varepsilon_{\alpha_{1}}+\varepsilon_{\alpha_{2}}-\varepsilon_{\alpha_{f}}-\varepsilon_{\alpha_{1}}\right)$, for diagram $1 \mathrm{~b}$ the index $\alpha_{1}$ runs over hole states and $\alpha_{2}$ over particles states $\left(\Delta E=\varepsilon_{\alpha_{f}}+\varepsilon_{\alpha_{1}}-\varepsilon_{\alpha_{1}}-\varepsilon_{\alpha_{2}}\right)$.

The results for single-hole states are obtained by (i) replacing the summations over intermediate particle (hole) states by summations over hole (particle) states, (ii) interchanging initial and final states and (iii) adding an overall minus sign.

The effective neutron $\left(t_{z}=\frac{1}{2}\right)$ and proton $\left(t_{z}=-\frac{1}{2}\right)$ charges are defined $\left.{ }^{12}\right)$ by

$$
e\left(j_{\mathrm{i}}, j_{\mathrm{f}} ; t_{z}\right) \equiv \frac{\Delta^{(1)}\left\langle j_{\mathrm{f}}\left(t_{z}\right)\left\|\boldsymbol{\Omega}^{(k)}\right\| j_{\mathrm{i}}\left(t_{z}\right)\right\rangle}{\left\langle j_{\mathrm{f}}\left(-\frac{1}{2}\right)\left\|\boldsymbol{\Omega}^{(k)}\right\| j_{\mathrm{i}}\left(-\frac{1}{2}\right)\right\rangle},
$$

where $\left\langle j_{\mathrm{f}}\left(-\frac{1}{2}\right) \| \boldsymbol{\Omega}^{(k)}|| j_{\mathrm{i}}\left(-\frac{1}{2}\right)\right\rangle$ represents the single-proton reduced transition matrix element

$$
\left\langle j_{\mathrm{f}}\left(t_{z}\right)\left\|\boldsymbol{\Omega}^{(k)}\right\| j_{\mathrm{i}}\left(t_{z}\right)\right\rangle=\frac{1}{\sqrt{ } 2}\left\langle\alpha_{\mathrm{f}}\left\|\left|\boldsymbol{\Omega}^{(k, 0)}\right|\right\| \alpha_{\mathrm{i}}\right\rangle+\frac{(-1)^{t_{z}+\frac{1}{2}}}{\sqrt{ } 6}\left\langle\alpha_{\mathrm{f}}\left\|\left|\boldsymbol{\Omega}^{(k, 1)}\right|\right\| \alpha_{\mathrm{i}}\right\rangle .
$$

\subsection{APPLICATIONS}

Eq. (1) has been applied to the calculation of corrections to the matrix elements of the electric quadrupole operator

$$
\boldsymbol{\Omega}^{(2)}=e r^{2} Y^{2}(\hat{r})\left(\frac{1}{2}-t_{z}\right)
$$

for single-patticle transitions between sd (and $\mathrm{fp}$ ) shell states. The two-body matrix 
elements of the Tabakin interaction required for the present calculation are obtained in the following way. First the matrix elements are expressed as linear combinations of the matrix elements in the relative coordinate system in the customary way with the use of Moshinsky brackets. The latter matrix elements are modified by the inclusion of the second-order Born corrections ${ }^{2,4}$ ). These corrections are due to the excitation of two nucleons to high-lying single-particle states and have been shown to be important for a calculation of energy levels ${ }^{2}$ ) in ${ }^{18} \mathrm{~F}$. The evaluation of these corrections for the Tabakin interaction has been described in detail in refs. ${ }^{2,5}$ ).

The intermediate states in eq. (1) have been restricted to $2 h \omega$ excitations, i.e. the hole states are taken in the $1 \mathrm{p}$ and $1 \mathrm{~d} 2 \mathrm{~s}$ shells and the particle states in the 1f $2 \mathrm{p}$ and $1 \mathrm{~g} 2 \mathrm{~d} 3 \mathrm{~s}$ shells, respectively.

The matrix elements of the E2 operator have been evaluated by the use of harmonicoscillator wave functions with size parameter $m \omega / h=0.286 \mathrm{fm}^{-2}$. The energy denominators are approximated by the value of $-2 \hbar \omega(\hbar \omega=12 \mathrm{MeV})$.

TAble 1a

Effective neutron charges for E2 transitions in the sd and fp shells

\begin{tabular}{|c|c|c|c|c|c|c|c|c|}
\hline & $d_{\frac{1}{2}}$ & $s_{\frac{1}{2}}$ & $d_{z}$ & & $f_{\frac{z}{3}}$ & $p_{z}$ & $f_{t}$ & $p_{t}$ \\
\hline $\begin{array}{l}d_{f} \\
s_{t} \\
d_{\frac{1}{2}}\end{array}$ & 0.64 & 0.66 & $\begin{array}{l}0.77 \\
0.55 \\
0.61\end{array}$ & $\begin{array}{l}f_{\frac{7}{2}} \\
p_{\frac{2}{2}} \\
f_{\frac{3}{2}}\end{array}$ & 0.50 & $\begin{array}{l}0.43 \\
0.34\end{array}$ & $\begin{array}{l}0.66 \\
0.32 \\
0.50\end{array}$ & $\begin{array}{l}0.38 \\
0.34\end{array}$ \\
\hline
\end{tabular}

TABLe 1b

Effective proton charges for $\mathrm{E} 2$ transitions in the sd and fp shells

\begin{tabular}{|c|c|c|c|c|c|c|c|c|}
\hline & $d_{2}$ & $s_{\frac{1}{2}}$ & $d_{\frac{\pi}{2}}$ & & $f_{\frac{z}{z}}$ & $\mathrm{p}_{\frac{1}{2}}$ & $f_{\frac{1}{2}}$ & $\mathrm{p}_{\frac{1}{2}}$ \\
\hline $\begin{array}{l}d_{\frac{1}{2}} \\
s_{\frac{1}{2}} \\
d_{\frac{1}{2}}\end{array}$ & 0.24 & 0.25 & $\begin{array}{l}0.34 \\
0.23 \\
0.27\end{array}$ & $\begin{array}{l}\mathrm{f}_{\frac{7}{2}} \\
\mathrm{p}_{\frac{3}{2}} \\
\mathrm{f}_{\frac{2}{2}}\end{array}$ & 0.20 & $\begin{array}{l}0.18 \\
0.14\end{array}$ & $\begin{array}{l}0.32 \\
0.13 \\
0.20\end{array}$ & $\begin{array}{l}0.16 \\
0.11\end{array}$ \\
\hline
\end{tabular}

The calculated effective proton and neutron charges are given in tables $1 \mathrm{a}$ and $\mathrm{b}$. It can be seen that the effective neutron charges are larger than the effective proton charges roughly by a factor of 2.5. This can be explained by the fact that the effective charge is due to proton excitations from the core. In the case of a proton valence particle, only $T=1$ nucleon-nucleon matrix elements can contribute to the effective charge; these are in general smaller than the $T=0$ matrix elements.

The rather large variations in the calculated effective charges do not support the assumption ${ }^{9,10}$ ) that the effective charge may be approximated by an overall constant effective charge independent of the configurations considered.

From an examination of the various contributions to the effective charges we find 
that they mainly derive from some particular particle-hole combinations $\mathrm{f}_{\frac{3}{2}} \mathrm{p}_{\frac{3}{2}}^{-1}$, $f_{t} p_{t}^{-1}, g_{\frac{t}{2}} d_{\frac{t}{t}}^{-1}$ or $g_{\frac{t}{2}} d_{t}^{-1}$. This appears to be caused both by larger radial overlaps in the two-body matrix elements and by larger matrix elements of the one-body operator.

It should be remarked that the corrections of the type considered above, cannot be expected to be the only contributions to the effective charge. For instance, the deformed many-particle-many-hole configurations will also contribute. In a recent ${ }^{13}$ ) calculation for the $\mathrm{Ca}$ region the admixing of these deformed (Nilsson) states with the spherical single-particle shell-model states has been evaluated explicitly. One should expect that the use of such mixed wave functions combined with the present effective charges will constitute a sensible comparison of the calculations with the experimental data.

Further corrections could be expected to come from iterations of the bubble diagrams of fig. 1. However, it is claimed ${ }^{12}$ ) that these corrections are quite small.

In a recent calculation ${ }^{19}$ ) very large contributions to the isoscalar effective charge were obtained from the RPA diagrams.

\subsection{ELECTRIC QUADRUPOLE MOMENTS OF ${ }^{80} \mathrm{~K}$ AND ${ }^{{ }^{\circ} \mathrm{K}}$}

The electric quadrupole moment of ${ }^{39} \mathrm{~K}$ has the experimental value $\left.{ }^{8}\right) 9 \pm 2 e \cdot \mathrm{fm}^{2}$. The value associated with the pure $1 \mathrm{~d}_{\frac{1}{2}}$ hole state, of course, depends on the choice of the $1 \mathrm{~d}_{\frac{1}{2}}$ radial wave function. If a harmonic-oscillator wave function is used, the zero-order quadrupole moment is found to be $4.9 e \cdot \mathrm{fm}^{2}$, whereas a Woods-Saxon wave function of comparable size yields $5.1 e \cdot \mathrm{fm}^{2}$.

Upon application of the effective $1 \mathrm{~d}_{\frac{3}{2}}$ proton charge as obtained in subsect. 2.2 , the value of the quadrupole moment only increases to $6.3 \mathrm{e} \cdot \mathrm{fm}^{2}$. Therefore it is likely that the remaining discrepancy should come from admixtures of two-particle-threehole states, which are ignored in the present calculation.

The electric quadrupole moment of ${ }^{40} \mathrm{~K}$ has the experimental value ${ }^{8}$ ) of $-9.3 \pm 2.5 e \cdot \mathrm{fm}^{2}$. If one assumes that the ground state of ${ }^{40} \mathrm{~K}$ is described by a pure $1 \mathrm{f}_{\frac{1}{2}} 1 \mathrm{~d}_{\frac{3}{2}}^{-1}$ configuration, one obtains a value of $-2.4 e \cdot \mathrm{fm}^{2}$. The wave function obtained from a recent diagonalization ${ }^{4}$ ) within the $1 \mathrm{h \omega}$ particle-hole space with the Tabakin interaction yields a value of $-4.4 e \cdot \mathrm{fm}^{2}$ for free neutron and proton charges. With the effective single-particle charges calculated in subsect. 2.2 , the value of the quadrupole moment becomes $-8.6 e \cdot \mathrm{fm}^{2}$.

In addition to the single-particle renormalization diagram, there are diagrams that may be considered to represent two-body renormalization processes. The relevant diagrams are shown in fig. 2.

It is seen that diagram 2 a contains exclusion-principle-violating terms as shown in fig. 3a. However, as the single-particle renormalization diagrams have been used, also the exclusion-principle-violating diagram $3 b$ has been in fact included. As the contributions of diagrams $3 a$ and $b$ cancel, all contributions of diagram $2 a$ (and similarly for diagram $2 \mathrm{c}$ ) must indeed be taken into account.

We note that other possible variants of these diagrams do not contribute in the 
${ }^{40} \mathrm{~K}$ case, as the E2 operator can act only on the proton line. Evaluation of fig. $2 \mathrm{a}$ yields

$$
\begin{aligned}
& \Delta^{(1)}\left\langle\alpha_{\mathrm{p}^{\prime}} \alpha_{\mathrm{h}^{\prime}}^{-1} ; \Gamma^{\prime} \|\left|\Omega^{(\Lambda)}\right| \mid \alpha_{\mathrm{p}} \alpha_{\mathrm{h}}^{-1} ; \Gamma\right\rangle \\
&=-\sum_{\alpha_{1} \Gamma} \frac{1}{\Delta E}(-1)^{\alpha_{\mathrm{p}}+\alpha_{1}}\left(2 \Gamma^{\prime \prime}+1\right)\left((2 \Gamma+1)\left(2 \Gamma^{\prime}+1\right)\right)^{\frac{1}{2}} \\
& \times\left(1+\delta_{1 \mathrm{p}^{\prime}}\right)^{\frac{1}{2}}\left\{\begin{array}{lll}
\Gamma^{\prime} & \Gamma & A \\
\alpha_{\mathrm{h}} & \alpha_{1} & \alpha_{\mathrm{p}}
\end{array}\right\}\left\{\begin{array}{lll}
\alpha_{1} & \alpha_{\mathrm{p}} & \Gamma^{\prime} \\
\alpha_{\mathrm{h}^{\prime}} & \alpha_{\mathrm{p}^{\prime}} & \Gamma^{\prime \prime}
\end{array}\right\} \\
& \times\left\langle\alpha_{\mathrm{p}^{\prime}} \alpha_{1} ; \Gamma^{\prime \prime}|V| \alpha_{\mathrm{p}} \alpha_{\mathrm{h}^{\prime}} ; \Gamma^{\prime \prime}\right\rangle\left\langle\alpha_{\mathrm{h}}||\left|\Omega^{(\Lambda)} \|\right| \alpha_{1}\right\rangle,
\end{aligned}
$$

where the index $\alpha_{1}$ runs over particle states; for fig. $2 \mathrm{~d}$ the index $\alpha_{1}$ runs over hole states, and an overall minus sign should be added to the right-hand side of eq. (2).

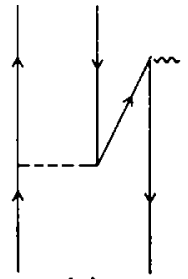

(a)

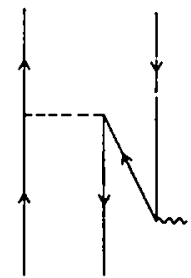

(b)

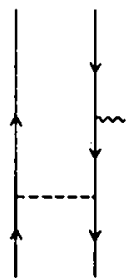

(c)

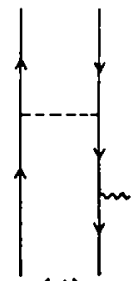

(d)

Fig. 2. First-order two-body renormalization diagrams for ${ }^{40} \mathrm{~K}$.

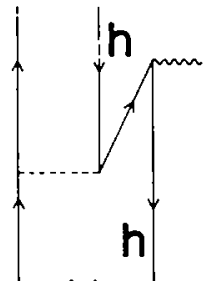

(a)

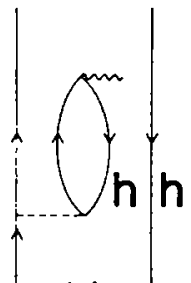

(b)

Fig. 3. Exclusion-principle-violating two-body diagrams for ${ }^{10} \mathrm{~K}$.

The contribution of fig. $2 \mathrm{~b}$ (and $2 \mathrm{c}$ ) can be obtained from expression (2) by appropriate changes of the labels on the right-hand side.

Again the energy denominators are taken to be $-2 \hbar \omega$.

The contributions of these two-body renormalization diagrams are relatively small because there exist only few intermediate states. The diagrams $2 a$ and $b$ with intermediate particle states contribute $+0.3 e \cdot \mathrm{fm}^{2}$, whereas the diagrams $2 \mathrm{c}$ and $\mathrm{d}$, with intermediate hole states contribute $-0.1 e \cdot \mathrm{fm}^{2}$.

Thus in the case of ${ }^{40} \mathrm{~K}$ the first-order corrections give already a satisfactory agreement with the experimental electric quadrupole moment.

This may indicate that the ${ }^{40} \mathrm{~K}$ ground state contains less deformed components than the ground state of ${ }^{39} \mathrm{~K}$. 


\section{Second-order corrections to magnetic dipole transitions and $f t$ values}

Thus far first-order corrections to matrix elements of the electric quadrupole operator have been considered. It is well known that there are no first-order corrections to the matrix elements of the magnetic dipole operator for the case of one nucleon outside a $j j$ and $L S$ closed shell.

Second-order calculations for the magnetic moments of $A=39$ and $A=41$ nuclei have previously been performed by Ichimura and Yazaki ${ }^{14}$ ), who used a phenomenological interaction, and Mavromatis, Zamick and Brown ${ }^{15,16}$ ), who used the (semi-realistic) Kallio-Kolltveit interaction.

The latter calculations gave a very satisfactory result for the magnetic moment of ${ }^{41} \mathrm{Ca}$; however, in the case of ${ }^{39} \mathrm{~K}$ the inclusion of second-order corrections on the Schmidt value for the magnetic moment resulted in an increased discrepancy with experiment.

In this section, we shall calculate second-order corrections to the magnetic moments of the same nuclei with the Tabakin interaction. Moreover, we shall investigate how the $l$-forbidden $2 s_{\frac{1}{2}} \rightarrow 1 d_{\frac{3}{3}}$ M1 transition will be enhanced by core polarization corrections. Finally, the effect of core polarization on the $f t$ values of the $1 \mathrm{f}_{\frac{z}{z}} \rightarrow 1 \mathrm{f}_{\frac{z}{z}}$ and $1 \mathrm{~d}_{f}^{-1} \rightarrow 1 \mathrm{~d}_{f}^{-1}$ beta transitions is studied.

\subsection{FORMALISM}

The second-order corrections to the reduced matrix elements of a single-particle operator which does not connect a single-particle state $|\alpha\rangle$ with any core-excited state, are given by

$$
\begin{aligned}
\Delta^{(2)}\left\langle\alpha_{\mathrm{f}}\left|\| \boldsymbol{\Omega}^{(\Lambda)}\right||| \alpha_{\mathrm{i}}\right\rangle= & \left\langle\alpha_{\mathrm{f}}\left\|\left|V \frac{P}{E-H_{0}} \boldsymbol{\Omega}^{(\Lambda)} \frac{P}{E-H_{0}} V \|\right| \alpha_{\mathrm{i}}\right\rangle\right. \\
& -\frac{1}{2}\left\langle\alpha_{\mathrm{f}}\left|V \frac{P}{\left(E-H_{0}\right)^{2}} V\right| \alpha_{\mathrm{f}}\right\rangle\left\langle\alpha_{\mathrm{f}}\left\|\left|\boldsymbol{\Omega}^{(\Lambda)}\right|\right\| \alpha_{\mathrm{i}}\right\rangle \\
& -\frac{1}{2}\left\langle\alpha_{\mathrm{i}}\left|V \frac{P}{\left(E-H_{0}\right)^{2}} V\right| \alpha_{\mathrm{i}}\right\rangle\left\langle\alpha _ { \mathrm { f } } \left|\left\|\boldsymbol{\Omega}^{(\Lambda)}\left|\| \alpha_{\mathrm{i}}\right\rangle,\right.\right.\right.
\end{aligned}
$$

where $P$ projects off the single-particle states $|\alpha\rangle$.

Expression (3) is easily obtained with the Rayleigh-Schrödinger perturbation expansion. The last two terms arise because the wave functions up to second order are not normalized to unity. The same result can be represented diagrammatically in terms of Brandow's linked valence expansion ${ }^{\dagger}$. The diagrams for the latter expansion are shown in fig. 4 . Loops around the valence lines in figs. $4 \mathrm{f}$ and $g$ indicate ${ }^{17}$ ) that these lines carry a factor $(-1)$.

Explicit expressions for the second-order corrections to the nuclear moments of nuclei with $j j$ and $L S$ closed shells plus (or minus) one nucleon are given in ref. ${ }^{15}$ ).

' See ref. ${ }^{17}$ ), eq. (7.43). 
Since we also need expressions for the non-diagonal matrix elements of the electromagnetic operators, the general formulae for these cases will be given. Diagrams $4 a$ and $\mathrm{c}$ contribute to eq. (3)

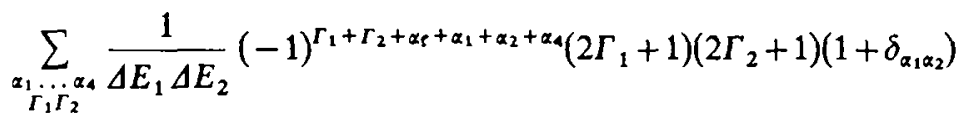

$$
\begin{aligned}
& \times\left(\left(1+\delta_{\mathrm{i} 4}\right)\left(1+\delta_{\mathrm{f} 4}\right)\right)^{ \pm}\left\{\begin{array}{ccc}
A & \Gamma_{2} & \Gamma_{1} \\
\alpha_{4} & \alpha_{\mathrm{i}} & \alpha_{\mathrm{f}}
\end{array}\right\}\left\{\begin{array}{ccc}
\Lambda & \alpha_{3} & \alpha_{2} \\
\alpha_{1} & \Gamma_{1} & \Gamma_{2}
\end{array}\right\} \\
& \times\left\langle\alpha_{1} \alpha_{2} ; \Gamma_{1}|V| \alpha_{\mathrm{i}} \alpha_{4} ; \Gamma_{1}\right\rangle\left\langle\alpha_{1} \alpha_{2} ; \Gamma_{2}|V| \alpha_{\mathrm{f}} \alpha_{4} ; \Gamma_{2}\right\rangle\left\langle\alpha_{3}||\left|\boldsymbol{\Omega}^{(\Lambda)}\right| \| \alpha_{2}\right\rangle .
\end{aligned}
$$

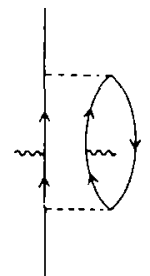

(a)

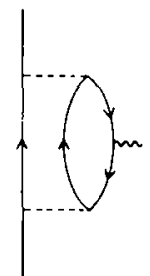

(b)

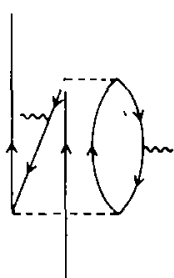

(c)

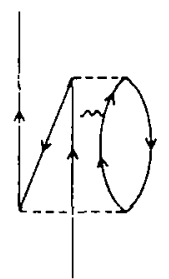

(d)

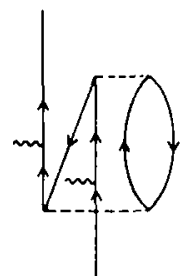

(e)

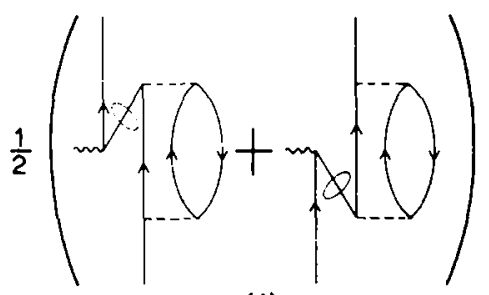

(f)

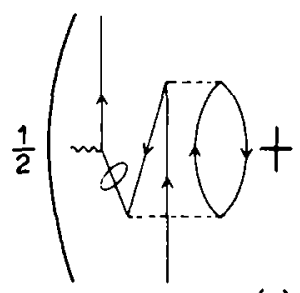

(g)

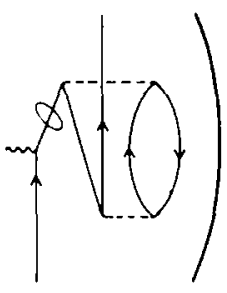

dipole operator.

For fig. 4a, the indices $\alpha_{1}, \alpha_{2}$ and $\alpha_{3}$ represent particle states and $\alpha_{4}$ a hole state, whereas for fig. $4 c$, the indices $\alpha_{1}, \alpha_{2}$ and $\alpha_{3}$ stand for holes states and $\alpha_{4}$ for a particle state.

Diagrams $4 \mathrm{~b}$ and $\mathrm{d}$ contribute

$$
\begin{aligned}
& -\frac{1}{2} \sum_{\alpha_{1} \ldots \alpha_{4} \Gamma} \frac{1}{\Delta E_{1} \Delta E_{2}}(2 \Gamma+1)(-1)^{\Gamma+\alpha_{3}+\alpha_{1}}\left(1+\delta_{\alpha_{1} \alpha_{2}}\right)\left(\left(1+\delta_{\mathrm{i} 3}\right)\left(1+\delta_{\mathrm{f} 4}\right)\right)^{\frac{1}{2}} \\
& \quad \times\left\{\begin{array}{lll}
\Lambda & \alpha_{4} & \alpha_{3} \\
\Gamma & \alpha_{\mathrm{i}} & \alpha_{\mathrm{f}}
\end{array}\right\}\left\langle\alpha_{1} \alpha_{2} ; \Gamma|V| \alpha_{\mathrm{i}} \alpha_{3} ; \Gamma\right\rangle\left\langle\alpha_{1} \alpha_{2} ; \Gamma|V| \alpha_{\mathrm{f}} \alpha_{4} ; \Gamma\right\rangle\left\langle\alpha_{3}||\left|\boldsymbol{\Omega}^{(\Lambda)}\right||| \alpha_{4}\right\rangle .
\end{aligned}
$$

For fig. $4 \mathrm{~b}$, the indices $\alpha_{1}$ and $\alpha_{2}$ represent particle states and $\alpha_{3}$ and $\alpha_{4}$ hole states, whereas for fig. $4 \mathrm{~d}$, the indices $\alpha_{1}$ and $\alpha_{2}$ represent hole states and $\alpha_{3}$ and $\alpha_{4}$ particle states.

The contribution from diagram $4 \mathrm{e}$ (which equals -2 times the contribution of diagram 
$4 \mathrm{~g}$ ) can be combined with the contributions from the folded diagrams $4 \mathrm{f}$ and $\mathrm{g}$ to give

$$
\begin{aligned}
& -\frac{1}{2}\left\{\sum_{\substack{\alpha_{1} \alpha_{2} \\
\alpha_{3} \Gamma}} \frac{1}{(\Delta E)^{2}} \frac{2 \Gamma+1}{2 \alpha_{\mathrm{i}}+1}\left(1+\delta_{\mathrm{i} 3}\right)\left(1+\delta_{\alpha_{1} \alpha_{2}}\right)\left\langle\alpha_{1} \alpha_{2} ; \Gamma|V| \alpha_{\mathrm{i}} \alpha_{3} ; \Gamma\right\rangle^{2}\right. \\
& \left.\quad+\sum_{\alpha_{1} \alpha_{2} \alpha_{3} \Gamma} \frac{1}{(\Delta E)^{2}} \frac{2 \Gamma+1}{2 \alpha_{\mathrm{f}}+1}\left(1+\delta_{\mathrm{f} 3}\right)\left(1+\delta_{\alpha_{1} \alpha_{2}}\right)\left\langle\alpha_{1} \alpha_{2} ; \Gamma|V| \alpha_{\mathrm{f}} \alpha_{3} ; \Gamma\right\rangle^{2}\right\}\left\langle\alpha_{\mathrm{f}}||\left|\boldsymbol{\Omega}^{(\Lambda)}\right||| \alpha_{\mathrm{i}}\right\rangle .
\end{aligned}
$$

The contribution from the one-nucleon excitation diagram $4 \mathrm{f}$ is obtained when $\alpha_{1}$ and $\alpha_{2}$ run over particle states and $\alpha_{3}$ over hole states; for the diagrams which involve two-nucleon excitations, $\alpha_{1}$ and $\alpha_{2}$ run over hole states and $\alpha_{3}$ over particle states.

Finally it should be remarked that for nuclei in which one nucleon is removed from a doubly closed shell, the proper results are obtained if (i) the summations previously restricted to particle (hole) states are now restricted to hole (particle) states, (ii) initial and final states are interchanged, and (iii) an overall minus sign is added.

\subsection{CALCULATION OF CORRECTIONS TO MAGNETIC DIPOLE MOMENTS}

The second-order corrections to the matrix elements of the magnetic dipole operator

$$
\mu=g_{i}^{(\mathrm{p})} l\left(\frac{1}{2}-t_{z}\right)+g_{s}^{(\mathrm{p})} s\left(\frac{1}{2}-t_{z}\right)+g_{s}^{(\mathrm{n})} s\left(\frac{1}{2}+t_{z}\right)
$$

are calculated separately for the spin and orbital parts. This is also useful since the spin part $\mu_{s}$ (the last two terms of the right-hand side) of the magnetic dipole operator is closely related to the Gamow-Teller operator in $\beta$-decay, for which corrections will be calculated below.

\begin{tabular}{|c|c|c|c|c|c|c|c|c|}
\hline & & $\begin{array}{l}\text { Zero } \\
\text { order }\end{array}$ & $\begin{array}{l}\text { Second-ord } \\
\text { two-particle } \\
\text { excitation }\end{array}$ & $\begin{array}{l}\text { der correction } \\
\text { one-particle } \\
\text { excitation }\end{array}$ & $\begin{array}{l}\text { total } \\
\text { correction }\end{array}$ & $\mu$ & $\mu_{\text {exp }}$ & $\begin{array}{c}\text { second-order } \\
\text { correction } \\
\text { MZB }{ }^{18} \text { ) }\end{array}$ \\
\hline${ }^{* 0} \mathrm{~K}$ & $\begin{array}{l}l \text {-part } \\
s \text {-part } \\
\mu\end{array}$ & $\begin{array}{r}1.80 \\
-1.68 \\
0.12\end{array}$ & $\begin{array}{r}-0.07 \\
0.03 \\
-0.04\end{array}$ & $\begin{array}{r}-0.21 \\
0.08 \\
-0.13\end{array}$ & $\begin{array}{r}-0.29 \\
0.12 \\
-0.17\end{array}$ & -0.05 & 0.39 & -0.26 \\
\hline${ }^{* 0} \mathrm{Ca}$ & $\begin{array}{l}l \text {-part } \\
s \text {-part } \\
\mu\end{array}$ & $\begin{array}{l}0.00 \\
1.15 \\
1.15\end{array}$ & $\begin{array}{r}0.08 \\
-0.03 \\
0.05\end{array}$ & $\begin{array}{r}0.18 \\
-0.03 \\
0.15\end{array}$ & $\begin{array}{r}0.26 \\
-0.06 \\
0.20\end{array}$ & 1.35 & & 0.26 \\
\hline${ }^{11} \mathrm{Ca}$ & $\begin{array}{l}l \text {-part } \\
s \text {-part } \\
\mu\end{array}$ & $\begin{array}{r}0.00 \\
-1.91 \\
-1.91\end{array}$ & $\begin{array}{l}0.11 \\
0.02 \\
0.13\end{array}$ & $\begin{array}{l}0.14 \\
0.05 \\
0.19\end{array}$ & $\begin{array}{l}0.25 \\
0.07 \\
0.32\end{array}$ & -1.59 & -1.60 & 0.28 \\
\hline${ }^{4} \mathrm{Sc}$ & $\begin{array}{l}l \text {-part } \\
s \text {-part } \\
\mu\end{array}$ & $\begin{array}{l}3.00 \\
2.79 \\
5.79\end{array}$ & $\begin{array}{l}-0.13 \\
-0.02 \\
-0.15\end{array}$ & $\begin{array}{l}-0.14 \\
-0.06 \\
-0.20\end{array}$ & $\begin{array}{l}-0.27 \\
-0.08 \\
-0.35\end{array}$ & 5.45 & & -0.28 \\
\hline
\end{tabular}

TABLE 2

Second-order contributions to magnetic dipole moments (n.m.) 
The various second-order contributions to the magnetic moments of ${ }^{39} \mathrm{~K},{ }^{39} \mathrm{Ca}$ ${ }^{41} \mathrm{Sc}$ and ${ }^{41} \mathrm{Ca}$ obtained with the use of eq. (3) are listed in table 2.

It is surprising to find that the corrections to the magnetic moment rather derive from contributions to the orbital part than to the spin part. The fact that almost all magnetic moments are found to lie inside the Schmidt values is usually ascribed to the fact that core polarization quenches the spin part. In the present case, this does not appear to be the most important effect. It was found that the contributions to the orbital part are mainly (for approximately $80 \%$ ) caused by excitations into intermediate states in which the $l$-operator acts on the $\lg _{9}$ or $\lg _{\frac{7}{2}}$ subshells. This indicates that the final correction is sensitive to the choice of the single-particle energies of the g-shell.

It is seen that in the case of $A=39$, an appreciable cancellation occurs between the corrections to the $s$ - and $l$-parts of the $\mu$ operator. The correction $(-0.17$ n.m.) to the Schmidt value of the moment of ${ }^{39} \mathrm{~K}(+0.12 \mathrm{n} . \mathrm{m}$. $)$ is somewhat smaller in magnitude than the correction obtained ${ }^{15}$ ) with the Kallio-Kolltveit interaction, but again it possesses the wrong sign (experimental value +0.39 n.m.).

Recently Chemtob ${ }^{17}$ ) has evaluated the contribution of the one-pion exchange currents to the magnetic dipole moments of ${ }^{39} \mathrm{~K}$ and ${ }^{41} \mathrm{Ca}$. For ${ }^{39} \mathrm{~K}$ he obtained a mesonic correction of 0.18 n.m., which largely removes the discrepancy. However, for ${ }^{41} \mathrm{Ca}$, the mesonic correction -0.18 n.m. re-introduces a deviation.

\subsection{CORRECTIONS TO THE $2 s_{\frac{1}{2}} \rightarrow 1 d_{\frac{3}{2}}$ l-FORBIDDEN MI TRANSITIONS}

In the sd shell, many $\mathrm{Ml}$ transitions are strongly inhibited because of the $l$-forbiddenness of the $s-d$ transition. We investigate to what extent second-order contributions may enhance the single-particle $2 \mathrm{~s}_{\frac{1}{2}} \rightarrow 1 \mathrm{~d}_{\frac{3}{2}}$ transition.

The corrections to the reduced transition probabilities for a single-particle (hole) transition

$$
B\left(\mathrm{M} 1 ; j_{\mathrm{i}} \rightarrow j_{\mathrm{f}}, t_{z}\right)=\frac{1}{2 j_{\mathrm{i}}+1}\left|\left\langle j_{\mathrm{f}}\left(t_{z}\right) \| \boldsymbol{\Omega}^{(1)}|| j_{\mathrm{i}}\left(t_{z}\right)\right\rangle\right|^{2},
$$

can be simply obtained from expression (3) for the correction to the reduced matrix elements. In this case, there is no contribution from fig. $4 \mathrm{f}$ or $\mathrm{g}$ since the zero-order M1 matrix element vanishes. Evaluation of the core polarization contribution for the $2 \mathrm{~s}_{\frac{1}{2}} \rightarrow 1 \mathrm{~d}_{\frac{2}{2}}$ proton transition yields a very small contribution $B\left(\mathrm{M} 1 ; 2 \mathrm{~s}_{\frac{1}{2}} \rightarrow 1 \mathrm{~d}_{\frac{2}{3}}\right.$, $\left.t_{z}=-\frac{1}{2}\right) \approx 0.0004 \mathrm{n} \cdot \mathrm{m} .^{2}$. A measurement of the lifetime and E2/M1 mixing ratio for the $\frac{1}{2}^{+} \rightarrow \frac{3}{2}^{+}$transition in ${ }^{39} \mathrm{~K}$ could provide a possible check on this correction (calculated width $\Gamma(\mathrm{M} 1)=70 \mu \mathrm{eV}$ ).

\subsection{BETA DECAY OF ${ }^{11} \mathrm{Sc}$ and ${ }^{\circ 0} \mathrm{Ca}$}

The $f t$ values ${ }^{18}$ ) for the $\beta$-decay of the ground states of ${ }^{41} \mathrm{Sc}$ and ${ }^{39} \mathrm{Ca}$ to the mirror nuclei are slightly larger than the values predicted by the single-particle model. 
The corrections to the squares of the Gamow-Teller matrix elements $\left(\tau_{x}=2 t_{x}\right)$

$$
\left|M_{\mathrm{GT}}\right|^{2}=\frac{1}{2 j_{\mathrm{i}}+1}\left|\left\langle j_{\mathrm{f}}\left( \pm \frac{1}{2}\right)\left\|\sigma \tau_{x}\right\| j_{\mathrm{i}}\left(\mp \frac{1}{2}\right)\right\rangle\right|^{2}
$$

can be obtained in terms of the second-order corrections to the spin part of the magnetic dipole operator.

It can be seen that

$$
\begin{aligned}
& \Delta^{(2)}\left\langle j_{\mathrm{r}}\left( \pm \frac{1}{2}\right)\left\|\sigma \tau_{x}\right\| j_{\mathrm{i}}\left(\mp \frac{1}{2}\right)\right\rangle \\
&= \pm \frac{2}{g_{s}^{(\mathrm{p})}-g_{s}^{(\mathrm{n})}} \Delta^{(2)}\left(\left\langle j_{\mathrm{f}}\left(\frac{1}{2}\right)\left\|\mu_{s}\right\| j_{\mathrm{i}}\left(\frac{1}{2}\right)\right\rangle-\left\langle j_{\mathrm{f}}\left(-\frac{1}{2}\right)\left\|\boldsymbol{\mu}_{s}\right\| j_{\mathrm{i}}\left(-\frac{1}{2}\right)\right\rangle\right) .
\end{aligned}
$$

For the ${ }^{39} \mathrm{Ca}$ decay, the value of $\left|M_{\mathrm{GT}}\right|^{2}$ for the single-particle $1 \mathrm{~d}_{\frac{3}{2}} \rightarrow 1 \mathrm{~d}_{\frac{3}{2}}$ transition is 0.60 . Inclusion of second-order corrections reduces the latter value to 0.52 . This yields an $f t$ value of $3526 \mathrm{~s}$ to be compared with the experimental value ${ }^{18}$ ) of $4150 \pm 300 \mathrm{~s}$.

For the ${ }^{41} \mathrm{Sc}$ decay, the value of $\left|M_{\mathrm{GT}}\right|^{2}$ for the single-particle transition is 1.29 . Second-order contributions reduce this value to 1.18 ; the corresponding $f t$ value is $2302 \mathrm{~s}$ to be compared with the experimental value ${ }^{18}$ ) of $2560 \pm 160 \mathrm{~s}$.

\section{Summary and conclusion}

It is one of the attractive features of realistic nuclear forces that no parameters are to be fitted to the spectroscopic data. One still has to take the single-particle energies from experiment; and also one assumes that the harmonic-oscillator wave functions provide a self-consistent basic set of radial wave functions. When the effect of core polarization is taken into account, the calculated level schemes near doubly closed shells are very encouraging. A more critical check consists in the calculation of nuclear electromagnetic properties. The results of the present paper show that in general the effects of core polarization improve the agreement with experiment. In the case of the magnetic dipole operator, at least some of the remaining discrepancies could be due to exchange-current effects that appear to be non-negligible. As has been remarked already, the renormalization of the $\mathrm{Ml}$ operator derives largely from the $l$-part of the operator. If one would introduce effective $g_{l}$ factors, the value of $g_{l}$ for a proton would be somewhat smaller than 1 and for a neution somewhat larger than 0 . However, the magnitude of the correction depends on the configuration involved.

As for the case of the electric quadrupole moments, it would be interesting to calculate all second-order contributions to the effective charges in order to investigate whether the rule-of-thumb effective charge of $0.5 e$ will be approached somewhat more closely. However, the number of second-order diagrams is appreciable.

Furthermore, it is worthwhile to study the problem of effective charges for nuclei far from closed shells. 
The authors would like to thank Professor P. M. Endt for reading the manuscript. This investigation was supported by the joint program of the "Stichting voor Fundamenteel Onderzoek der Materie" and the "Nederlandse Organisatie voor Zuiver Wetenschappelijk Onderzoek".

\section{References}

1) T. T. S. Kuo, Nucl. Phys. A90 (1967) 199

2) D. Clement and E. Baranger, Nucl. Phys. A108 (1968) 27

3) F. Tabakin, Ann. of Phys. 30 (1964) 51

4) A. E. L. Dieperink, H. P. Leenhouts and P. J. Brussaard, Nucl. Phys. A106 (1968) 556

5) D. Clement and E. Baranger, Nucl. Phys. A120 (1968) 25

6) A. K. Kerman and M. K. Pal, Phys. Rev. 162 (1967) 970

7) P. M. Endt and C. van der Leun, Nucl. Phys. A105 (1967) 1

8) V.S. Shirley, in Hyperfine structure and nuclear radiations, ed. by E. Matthias and D. A. Shirley, (North-Holland Publ. Co., Amsterdam, 1968)

9) E. C. Halbert, J. B. McGrory and B. H. Wildenthal, Phys. Lett. 20 (1968) 1112

10) A. P. Zucker, B. Buck and J. B. McGrory, Phys. Rev. Lett. 21 (1968) 39

11) A. de-Shalit and I. Talmi, Nuclear shell theory (Academic Press, New York, 1963)

12) M. Gmitro et al., Phys. Rev. Lctt. 20 (1968) 1185; Phys. Rev. 175 (1968) 1243

13) W. J. Gerace and A. M. Green, Nucl. Phys. A93 (1967) 110

14) M. Ichimura and K. Yazaki, Nucl. Phys. 63 (1965) 401

15) H. A. Mavromatis, L. Zamick and G. E. Brown, Nucl. Phys. 80 (1966) 545

16) H. A. Mavromatis and L. Zamick, Nucl. Phys. A104 (1967) 17

17) M. Chemtob, Nucl. Phys. A123 (1968) 449

18) E. J. Konopinski, The theory of beta radioactivity (Clarendon Press, Oxford, 1966) p. 151

19) S. Siegel and L. Zamick, Phys. Lett. 28B (1969) 453 